\title{
Caracterização da seca histórica da década de 2010 na Bacia do Rio Paraíba do Sul, Estado de São Paulo, Brasil
}

\author{
Characterization of the 2010-decade historical drought \\ in Paraiba do Sul River Basin, São Paulo State, Brazil
}

Anderson de Oliveira Neves ${ }^{1} \odot$, Mateus Ricardo Nogueira Vilanova ${ }^{2 *}$ (])

\begin{abstract}
RESUMO
A Bacia Hidrográfica do Rio Paraíba do Sul foi afetada por uma das secas mais severas de sua história, durante os anos de 2013 a 2015, que resultou em várias consequências para o gerenciamento de seus recursos hídricos. O objetivo deste artigo foi contribuir com o entendimento desse evento histórico, no trecho Paulista da Bacia do Rio Paraíba do Sul, por meio do cálculo e da análise do índice padronizado de precipitação. Esse índice foi calculado nas escalas de 1, 3, 6 e 12 meses, para 22 estações pluviométricas Iocalizadas na área de estudo. Os resultados demonstraram que a seca ocorrida entre 2013 e 2014 foi uma das mais severas já registradas, e que o verão de 2014 foi o período crítico no que diz respeito à redução da chuva regional. Essa seca impactou o armazenamento e a capacidade de regularização do principal reservatório da região, o Reservatório de Paraibuna. Embora o pico da crise hídrica tenha ocorrido, principalmente, em razão da seca de 2013/2014, verificou-se que ele foi influenciado pelo efeito cumulativo de uma seca anterior, ocorrida desde o início do ano de 2011. Esse resultado reforça a importância do planejamento plurianual da operação do Sistema Hidráulico da Bacia do Rio Paraíba do Sul.

Palavras-chave: chuva; índice padronizado de precipitação; escassez hídrica; Rio Paraíba do Sul.
\end{abstract}

\section{INTRODUÇÃO}

Segundo o Glossário Internacional de Hidrologia (WORLD METEOROLOGICAL ORGANIZATION; UNITED NATIONS EDUCATIONAL SCIENTIFIC AND CULTURAL ORGANIZATION, 2012), seca é uma "ausência prolongada ou deficiência acentuada de precipitação.”. Essa definição é consistente com a categoria de "seca meteorológica" apresentada por Mishra e Singh (2010), segundo

\begin{abstract}
The Paraiba do Sul River Basin was affected by one of the most severe drought periods in its recent history, during the years 2013 to 2015 , which resulted in several consequences for the management of its water resources. This article aims to contribute to the understanding of this historical event in the São Paulo Stretch of the Paraiba do Sul River Basin, through the calculation and analysis of the standardized precipitation index (SPI). SPI was calculated for the time scales of 1, 3, 6, and 12 months, for 22 rainfall stations located in the study area. The results showed that the drought occurred between 2013 and 2014 was one of the most severe ever recorded, and that the summer of 2014 was the critical period in terms of reduction of regional rainfall. This drought impacted the storage and regularization capacity of the main reservoir of the region, the Paraibuna Reservoir. Although the peak of the water crisis occurred mainly due to the 2013/2014 drought, it was found that it was influenced by the cumulative effect of a previous dry period, that occurred since the beginning of the year of 2011. This result reinforces the importance of multiannual planning for the operation of the Paraíba do Sul River Basin Hydraulic System.
\end{abstract}

Keywords: rainfall; standardized precipitation index; water shortage; Paraiba do Sul River. os quais a precipitação é a principal variável hidrológica utilizada na análise de secas meteorológicas. No Brasil, secas são consideradas desastres naturais climáticos, código 1.4.1.2.0 da Classificação e Codificação Brasileira de Desastres (COBRADE) (BRASIL, 2014).

A Bacia Hidrográfica do Rio Paraíba do Sul (BHRPS) é uma importante unidade hidrológica e de gerenciamento de recursos hídricos do

'Laboratório Associado de Combustão e Propulsão, Instituto Nacional de Pesquisas Espaciais - Cachoeira Paulista (SP), Brasil.

2Departamento de Engenharia Ambiental, Instituto de Ciência e Tecnologia, Universidade Estadual Paulista "Júlio de Mesquita Filho" - São José dos Campos (SP), Brasil.

*Autor correspondente: mateus.vilanova@unesp.br

Conflitos de interesse: os autores declaram não haver conflito de interesses.

Financiamento: nenhum.

Recebido: 25/01/2O19. Aceito: O2/O3/2O2O. Reg. ABES: 20190023 
Brasil, localizada em uma área altamente desenvolvida entre as Regiões Metropolitanas de São Paulo e Rio de Janeiro. Alguns sistemas de recursos hídricos emblemáticos para o Brasil (incluindo transposições de bacia e reservatórios hidrelétricos) localizam-se na BHRPS. Este artigo tem como foco a Porção Paulista da Bacia Hidrográfica do Rio Paraíba do Sul (SPBHRPS), que compõe a Unidade de Gerenciamento de Recursos Hídricos 2 (UGRHI-02) do Sistema Integrado de Gerenciamento dos Recursos Hídricos do Estado de São Paulo (SIGRH).

Durante a primeira metade da década de 2010, a Região Sudeste do Brasil foi afetada por uma severa escassez hídrica, resultado de uma quantidade de chuva muito inferior às médias históricas (COELHO et al., 2016; ESCOBAR, 2015). O pico dessa crise hídrica ocorreu entre os anos de 2014 e 2015, durante os quais o armazenamento de água de importantes sistemas de recursos hídricos (como o Sistema Canteira, responsável pelo abastecimento de parte da Região Metropolitana de São Paulo) atingiu níveis históricos de deplecionamento. As consequências dessa escassez incluíram a redução da geração hidrelétrica e da produção industrial, além do racionamento doméstico. A Figura 1 apresenta a evolução do volume útil do Reservatório de Paraibuna, o principal da BHRPS, durante esse período. Como pode ser visto na Figura 1, o reduzido volume de chuvas na BHRPS desde o final de 2013 resultou em um deplecionamento extremo do Reservatório de Paraibuna, que culminou com a marca de $0 \%$ de volume útil em janeiro de 2015.

Analisar as características de secas é uma tarefa fundamental para subsidiar a tomada de decisões no gerenciamento de recursos hídricos. Uma das principais ferramentas para a análise de secas são os índices de seca (IS). Um IS é uma "variável primordial para avaliar o efeito e definir diferentes parâmetros de uma seca, que incluem intensidade, duração, severidade e extensão espacial" (MISHRA; SINGH, 2010, p. 207). De acordo com Sayers et al. (2017), o uso de indicadores bem estabelecidos, como uma abordagem de gerenciamento de secas, iniciou-se em decorrência de grande secas ocorridas entre as décadas de 1930 e 1970. O índice padronizado de precipitação (IPP) (MCKEE; DOESKEN; KLEIST, 1993) é um dos IS mais utilizados (e.g., BONACCORSO et al., 2015; CALOIERO, 2017; ŁABȨDZKI, 2017; YAN et al., 2017). A grande aceitação do

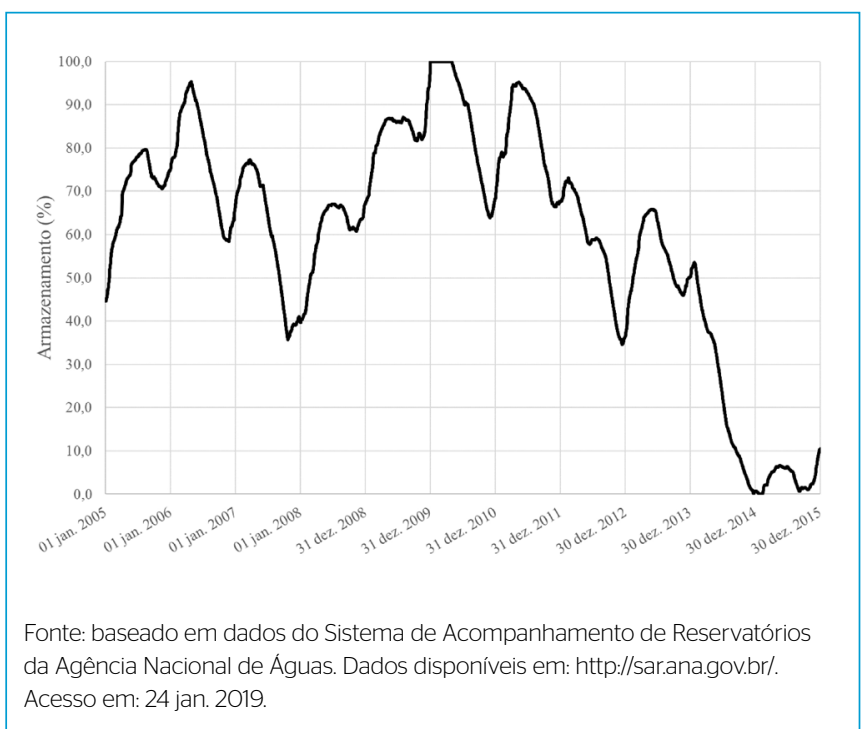

Figura 1 - Evolução do volume útil do Reservatório de Paraibuna entre 2005 e 2015.
IPP pode ser atribuída à sua simplicidade e ao fato de serem necessários apenas dados de precipitação para o seu cálculo (TSAKIRIS; VANGELIS, 2004). Apesar dessa simplicidade, o IPP permite comparar secas de diferentes locais (TSAKIRIS; VANGELIS, 2004) e analisar diferentes escalas temporais de seca (MISHRA; SINGH, 2010).

A singularidade da seca ocorrida na década de 2010, associada à importância da SPBHRPS no cenário brasileiro de recursos hídricos, motivou o desenvolvimento desta pesquisa, cujo objetivo foi contribuir com o entendimento desse evento histórico e suas consequências, por meio do cálculo e da análise do IPP para diversas estações pluviométricas localizadas na SPBHRPS. São objetivos específicos do trabalho: classificar a seca histórica da década de 2010 quanto à sua intensidade e determinar se esse foi o período mais seco dentre os registros de precipitação disponíveis; identificar quais regiões/cidades da SPBHRPS foram mais afetadas pela seca; e analisar a evolução temporal da chuva/seca na área de estudo, sobretudo no período de crise hídrica.

\section{METODOLOGIA}

A área da SPBHRPS (Figura 2) é de $14.444 \mathrm{~km}^{2}$, e sua vazão média de longo termo é de $216 \mathrm{~m}^{3} . \mathrm{s}^{-1}$ (GOVERNO DO ESTADO DE SÃO PAULO; FUNDO ESTADUAL DE RECURSOS HÍDRICOS; COMITÊ DAS BACIAS HIDROGRÁFICAS DO RIO PARAÍBA DO SUL, 2016).

O Sistema Hidráulico do Rio Paraíba do Sul (SHRPS) é um dos mais importantes do cenário brasileiro de recursos hídricos, uma vez que fornece água para o Vale do Rio Paraíba do Sul (uma das regiões mais ricas e industrializadas do Brasil) e para a Região Metropolitana do Rio de Janeiro (por meio de um sistema de bombeamento que transpõe águas da BHRPS para a Bacia do Rio Guandu). Por consequência da crise hídrica da década de 2010, a BHRPS foi interligada, também, ao Sistema Cantareira. O SHRPS, no Estado de São Paulo, é composto de quatro reservatórios (Tabela 1): Paraibuna, Santa Branca, Jaguari e Funil, e esse último se localiza majoritariamente no Estado do Rio de Janeiro, fora dos limites da UGRHI-02/SPBHRPS.

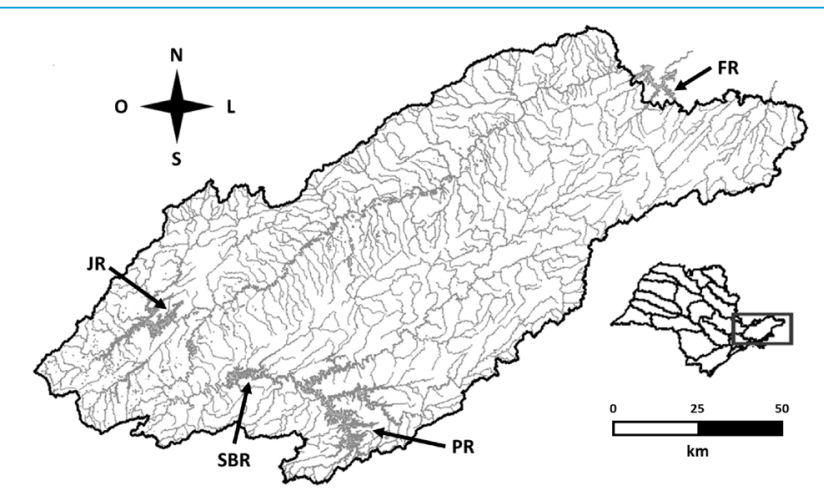

PR: Paraibuna; SBR: Santa Branca; JR: Jaguari; FR: Funil.

Fonte: adaptado de http://www3.funcate.org.br/geo/available/geoweb-v01CBHPS/src/php/app.php. Acesso em: 24 jan. 2019

Figura 2 - Porção Paulista da Bacia Hidrográfica do Rio Paraíba do Sul, Unidade de Gerenciamento de Recursos Hídricos 2. As linhas cinzas representam a hidrografia da bacia, e os polígonos, seus principais reservatórios: Paraibuna, Santa Branca, Jaguari e Funil. 
Tabela 1 - Características dos reservatórios do Sistema Hidráulico do Rio Paraíba do Sul.

\begin{tabular}{l|c|c|c} 
Reservatório & $\begin{array}{c}\text { Volume } \\
\text { máximo }\left(\mathrm{hm}^{3}\right)\end{array}$ & $\begin{array}{c}\text { Volume } \\
\text { útil }\left(\mathrm{hm}^{3}\right)\end{array}$ & $\begin{array}{c}\text { Capacidade } \\
\text { hidrelétrica (MW) }\end{array}$ \\
\hline Paraibuna & $4.731,7$ & $2.636,1$ & 86 \\
\hline Santa Branca & 439,0 & 308,0 & 59 \\
\hline Jaguari & $1.235,6$ & 792,5 & 28 \\
\hline Funil & 888,3 & 605,3 & 222 \\
\hline
\end{tabular}

Fonte: ANA (2017)

Considerando as 22 estações pluviométricas utilizadas neste estudo (vide Tabela 2), a precipitação anual média da região é de $1.330 \mathrm{~mm}$. O clima da região varia de Cwa a Aw e Am, segundo a classificação de Köppen (SÃO PAULO, 2011).

\section{Dados pluviométricos}

Este trabalho tem como foco a análise do período úmido e do ano hidrológico típico do Sudeste Brasileiro. Essa delimitação ocorreu em função das perspectivas operacionais e de gerenciamento do sistema de recursos hídricos da SPBHRPS, associada ao balanço hídrico sazonal e à variabilidade da precipitação regional. É evidente que anomalias de precipitação podem afetar mais intensamente o armazenamento e a operação do SHRPS caso ocorram durante o período úmido, quando é esperada a reposição dos volumes consumidos, majoritariamente, durante o período seco. Nesse sentido, o período úmido típico do Sudeste Brasileiro engloba a primavera (com início em 22 de setembro) e o verão (com início em 21 de dezembro). Em termos práticos (para a análise das séries pluviométricas e cálculo dos IPPs), foi considerado o início da primavera em $1^{\circ}$ de outubro e o término em 31 de dezembro, e o início do verão em $1^{\circ}$ de janeiro e o término em 31 de março. Com base nessa premissa, o período úmido inicia-se em outubro do ano $_{n}$ e termina em março do ano ${ }_{n+1}$; da mesma maneira, o ano hidrológico se inicia em outubro do ano ${ }_{n}$ e termina em setembro do ano $_{\mathrm{n}+1}$.

As séries de dados pluviométricos foram preparadas por Neves (2016), de acordo com a seguinte metodologia: séries de totais acumulados mensais de precipitação foram obtidas na base Hidroweb da Agência Nacional de Águas. Foi elaborado um inventário das estações pluviométricas localizadas sobre a área de estudo, resultando em 370 estações. Após o inventário, essas estações foram submetidas a um processo de triagem, para selecionar as séries de dados que poderiam ser utilizadas. Os critérios de triagem foram:

- Existência de pelo menos 30 anos de dados mensais de precipitação, retroativos a setembro de 2013 (referente ao início do período úmido de 2013/2014, durante o qual a seca se tornou evidente);

- Existência de dados para os anos de 2014 e 2015, durante os quais ocorreu o pico da crise hídrica;

- Integridade das séries superior a $80 \%$.

A integridade das séries de dados foi calculada dividindo o tamanho da amostra no período considerado pelo tamanho da série completa no mesmo período, caso não houvesse falhas de dados. Um inconveniente resultante do processo de triagem foi a inexistência de estações pluviométricas localizadas à montante do Reservatório de Paraibuna (na região de cabeceira dos rios Paraibuna e Paraitinga) que atendessem aos critérios estabelecidos. As estações selecionadas durante a triagem foram, então, consideradas estações base para o estudo.

No caso da existência de falhas nas séries de dados, foi realizado o preenchimento das falhas utilizando estações auxiliares. Essas estações foram selecionadas de acordo com a sua correlação com as estações base, que deveria ser superior a 0,85 . O preenchimento das falhas ocorreu por meio das equações obtidas por regressão linear simples entre as séries de dados das estações base e de suas respectivas auxiliares.

A Tabela 2 apresenta as principais características das estações base. Conforme a Tabela 2, a precipitação anual média na área de estudo varia de $1.066 \mathrm{~mm}$ em Taubaté a $1.827 \mathrm{~mm}$ em Monteiro Lobato, com valor médio de $1.330 \mathrm{~mm}$.

\section{Índice padronizado de precipitação}

O IPP é calculado tradicionalmente utilizando a distribuição gama. Essa distribuição é útil para séries hidrológicas assimétricas (CHOW; MAIDMENT; MAYS, 1988), sendo, geralmente, bem-sucedida na representação de dados de chuva, desde a escala diária até a anual (NAGHETTINI; PINTO, 2007). A função densidade da distribuição gama é obtida por meio da Equação 1.

$f_{g}(x)=\frac{x^{\alpha-1}}{\beta^{\alpha} \Gamma(\alpha)} \cdot e^{\frac{-x}{\beta}}$

Em que:

$\mathrm{f}_{\mathrm{g}}(\mathrm{x})=$ função densidade de probabilidade da distribuição gama;

$\mathrm{x}=$ acumulado de chuva no período de tempo considerado (mês, trimestre, semestre), em mm;

$\alpha$ e $\beta$ = parâmetros da distribuição;

$\mathrm{G}=$ função gama.

Os estimadores de máxima verossimilhança para $\alpha$ e $\beta$ são dados pelas Equações 2 e 3 (TIGKAS; VANGELIS; TSAKIRIS, 2015):

$\alpha=\frac{\left(1+\sqrt{1+\frac{4 \cdot\left(\ln (\bar{x})-\frac{\sum \ln (x)}{n}\right)}{3}}\right)}{4 \cdot\left(\ln (\bar{x})-\frac{\sum \ln (x)}{n}\right)}$

$\beta=\frac{\bar{x}}{\alpha}$

Em que:

$\bar{x}=$ média da chuva para o período considerado, em $\mathrm{mm}$;

$\mathrm{n}=$ tamanho da amostra

A função gama é indefinida para $\mathrm{x}=0$, sendo necessária a conversão da sua função cumulativa $\mathrm{F}(\mathrm{x})$ em $\mathrm{H}(\mathrm{x})$ (Equação 4):

$H(x)=q+(1-q) \cdot F(x)$

Em que:

$\mathrm{q}$ = probabilidade de ocorrência de uma chuva com valor "zero" (pode ser estimado dividindo o número de "zeros" da série por $\mathrm{n}$ );

$\mathrm{F}(\mathrm{x})$ = função cumulativa da distribuição gama.

$\mathrm{H}(\mathrm{x})$ = transformada na variável normal padronizada "Z", com média "zero" e desvio padrão "um” (TIGKAS; VANGELIS; TSAKIRIS, 2015). 
Tabela 2 - Características das estações base selecionadas.

\begin{tabular}{|c|c|c|c|c|c|c|}
\hline $\begin{array}{l}\text { Código } \\
\text { ANA }\end{array}$ & Cidade & Latitude & Longitude & $\begin{array}{l}\text { Altitude } \\
\text { (m) }\end{array}$ & $\begin{array}{c}\text { Ano de } \\
\text { início da série }\end{array}$ & $\begin{array}{l}\text { Precipitação anual média } \\
\text { (mm) }\end{array}$ \\
\hline 2244001 & Queluz & $22^{\circ} 32^{\prime} 58^{\prime \prime} \mathrm{S}$ & $44^{\circ} 47^{\prime} 20^{\prime \prime} \mathrm{W}$ & 500 & 1957 & 1.395 \\
\hline 2244010 & Cachoeira Paulista & $22^{\circ} 411^{\prime \prime} \mathrm{S}$ & $44^{\circ} 58^{\prime} 30^{\prime \prime} \mathrm{W}$ & 550 & 1936 & 1.382 \\
\hline 2245020 & Lorena & $22^{\circ} 44^{\prime} 00^{\prime \prime} \mathrm{S}$ & $45^{\circ} \mathrm{O} 5^{\prime} \mathrm{OO} \mathrm{O}^{\prime \prime} \mathrm{W}$ & 540 & 1957 & 1.276 \\
\hline 2245032 & Guaratinguetá & $22^{\circ} 48^{\prime} 44^{\prime \prime} \mathrm{S}$ & $45^{\circ} 10^{\prime} 57^{\prime \prime} \mathrm{W}$ & 519 & 1930 & 1.384 \\
\hline 2245035 & Pindamonhangaba & $22^{\circ} 49^{\prime} 00^{\prime \prime} \mathrm{S}$ & $44^{\circ} 22^{\prime} \mathrm{OO} " \mathrm{~W}$ & 540 & 1957 & 1.212 \\
\hline 2245048 & Pindamonhangaba & $22^{\circ} 54^{\prime} 40^{\prime \prime} \mathrm{S}$ & $45^{\circ} 28^{\prime} 13^{\prime \prime} \mathrm{W}$ & 524 & 1932 & 1.232 \\
\hline 2245053 & Aparecida & $22^{\circ} 57^{\prime} 00^{\prime \prime} \mathrm{S}$ & $45^{\circ} 15^{\prime} 00^{\prime \prime} \mathrm{W}$ & 690 & 1957 & 1.249 \\
\hline 2245054 & Monteiro Lobato & $22^{\circ} 56^{\prime} 00^{\prime \prime} \mathrm{S}$ & $45^{\circ} 50^{\prime} 00^{\prime \prime} \mathrm{W}$ & 680 & 1939 & 1.827 \\
\hline 2345001 & Taubaté & $23^{\circ} \mathrm{O} 2^{\prime} \mathrm{OO} " \mathrm{~S}$ & $45^{\circ} 34^{\prime} 00^{\prime \prime} \mathrm{W}$ & 610 & 1963 & 1.345 \\
\hline 2345004 & Caçapava & $23^{\circ} \mathrm{O} 2^{\circ} \mathrm{OO} " \mathrm{~S}$ & $45^{\circ} 477^{\prime} \mathrm{O}$ " W & 580 & 1969 & 1.549 \\
\hline 2345008 & Caçapava & $23^{\circ} 05^{\prime} 00^{\prime \prime} \mathrm{S}$ & $45^{\circ} 40^{\prime} 00^{\prime \prime} \mathrm{W}$ & 580 & 1945 & 1.173 \\
\hline 2345012 & Caçapava & $23^{\circ} 08^{\prime} 00^{\prime \prime} \mathrm{S}$ & $45^{\circ} 43^{\prime} 00^{\prime \prime} \mathrm{W}$ & 620 & 1942 & 1.282 \\
\hline 2345022 & Jambeiro & $23^{\circ} 15^{\circ} \mathrm{OO} 0^{\prime \prime} \mathrm{S}$ & 4541'OO" W & 700 & 1943 & 1.284 \\
\hline 2345062 & Taubaté & $23^{\circ} \mathrm{O} 222^{\prime \prime} \mathrm{S}$ & $45^{\circ} 33^{\prime} 32 ”$ W & 586 & 1937 & 1.290 \\
\hline 2345063 & Caçapava & $23^{\circ} \mathrm{O} 440^{\prime \prime} \mathrm{S}$ & $45^{\circ} 42^{\prime} 37^{\prime \prime} \mathrm{W}$ & 545 & 1929 & 1.290 \\
\hline 2345161 & Taubaté & $23^{\circ} \mathrm{O} 4^{\prime} \mathrm{OO} " \mathrm{~S}$ & $45^{\circ} 30^{\prime} 00^{\prime \prime} \mathrm{W}$ & 600 & 1957 & 1.066 \\
\hline 2345164 & São José dos Campos & $23^{\circ} 11^{\prime} 00^{\prime \prime} \mathrm{S}$ & $45^{\circ} 48^{\prime} \mathrm{OO}$ " W & 570 & 1960 & 1.275 \\
\hline 2346019 & Santa Isabel & $23^{\circ} 20^{\prime} 00^{\prime \prime} \mathrm{S}$ & $46^{\circ} 14^{\prime} \mathrm{OO}$ " W & 690 & 1937 & 1.345 \\
\hline \multirow[t]{2}{*}{2346344} & Igaratá & $23^{\circ} 12^{\prime} \mathrm{OO}$ " S & $46^{\circ} 09^{\prime} 00^{\prime \prime} \mathrm{W}$ & 780 & 1972 & 1.409 \\
\hline & & & & & Média & 1.330 \\
\hline
\end{tabular}

Fonte: elaborado pelos autores.

$\mathrm{O}$ valor de $\mathrm{Z}$ corresponde ao valor do IPP. Uma normalização computacional dessa função é comumente utilizada por meio da aproximação proposta por Abramowitz e Stegun (1965), conforme as Equações de 5 a 8 (EDWARDS; MCKEE, 1997).

$Z=I P P=-\left(t-\frac{c_{0}+c_{1} t+c_{2} t^{2}}{1+d_{1} t+d_{2} t^{2}+d_{3} t^{3}}\right)$ para $0<\mathrm{H}(\mathrm{x}) £ 0,5$

$Z=I P P=+\left(t-\frac{c_{0}+c_{1} t+c_{2} t^{2}}{1+d_{1} t+d_{2} t^{2}+d_{3} t^{3}}\right)$ para $0,5<\mathrm{H}(\mathrm{x})<1$

$t=\sqrt{\ln \left(\frac{1}{(H(x))^{2}}\right)}$ para $0<\mathrm{H}(\mathrm{x}) £ 0,5$

$t=\sqrt{\ln \left(\frac{1}{(1-H(x))^{2}}\right)}$ para $0,5<\mathrm{H}(\mathrm{x})<1$

Em que:

t = variável da aproximação de Abramowitz e Stegun (1965);

$c_{0}, c_{1}, c_{2}, d_{1}, d_{2}, d_{3}=$ parâmetros da aproximação.

Nas Equações 5 e 6, os valores das constantes são: $c_{0}=2,515517, c_{1}=0,802853$, $c_{2}=0,010328, d_{1}=1,432788, d_{2}=0,189269$ e d $d_{3}=0,001308$.

A Tabela 3 apresenta a classificação de secas conforme os valores do IPP, segundo os padrões propostos por McKee, Doesken e Kleist (1993) e pela
Tabela 3 - Classificação de secas conforme os valores do índice padronizado de precipitação.

\begin{tabular}{l|c|c}
$\mathrm{IPP}$ & Classe de seca & Severidade, tempo de retorno (anos) \\
\hline $\mathrm{O}>\mathrm{IPP}^{3}-0,99$ & Branda & 3 \\
\hline$--^{3} \mathrm{IPP}^{3}-1,49$ & Moderada & 10 \\
\hline$-1,5 \mathrm{O}^{3} \mathrm{IPP}^{3}-1,99$ & Severa & 20 \\
\hline$-2 \leq \mathrm{IPP}$ & Extrema & 50
\end{tabular}

IPP: índice padronizado de precipitação.

Fonte: McKee, Doesken e Kleist (1993) e Svoboda et al. (2012).

Organização Meteorológica Mundial (SVOBODA et al., 2012). Alguns autores (e.g., SVOBODA et al., 2012) consideram valores do IPP entre 0,99 e -0,99 como condições próximas à normalidade. De acordo com a proposta original do IPP (MCKEE; DOESKEN; KLEIST, 1993), uma seca inicia-se dentro de uma série temporal toda vez que o valor do IPP se torna negativo, e termina no momento subsequente, quando esse valor se torna "zero". Os IPPs foram calculados para quatro escalas temporais (1, 3, 6 e 12 meses) e para quatro períodos: primavera, verão, período úmido e ano hidrológico. Os cálculos foram realizados por meio do aplicativo DrinC (TIGKAS; VANGELIS; TSAKIRIS, 2015).

A apresentação dos resultados deste trabalho tem como foco secas moderadas, severas e extremas, ou seja, valores de IPP iguais ou menores a $-1,00$. Condições próximas à normalidade/secas brandas $\left(0>\mathrm{IPP}^{3}-0,99\right)$ foram consideradas apenas para definir os momentos de início e término dos eventos 
de seca. Considerando o gerenciamento e a operação do Sistema de Recursos Hídricos da SPBHRPS, foram destacados os resultados do período posterior a 2004, que engloba os 10 anos anteriores ao pico da crise hídrica, apesar de todos os IPP terem sido calculados para o período completo das estações base. Nas análises regionais, foram considerados IPPs médios, calculados a partir das médias aritméticas das estações pluviométricas utilizadas. Não foram utilizadas técnicas para a interpolação espacial dos resultados em razão da falta de cobertura de estações pluviométricas em grandes áreas da SPBHRPS, sobretudo em suas regiões limítrofes (Serras da Mantiqueira e do Mar/Bocaina), bem como na já mencionada região das nascentes dos rios Paraibuna e Paraitinga.

\section{RESULTADOS E DISCUSSÃO}

A Figura 3 apresenta a frequência regional de secas para cada escala temporal analisada. Essa frequência de secas foi calculada dividindo o número de estações pluviométricas que apresentaram secas (IPP $\leq-1$ ) pelo número total de estações estudadas (22 estações). A Figura 4 apresenta o IPP médio das

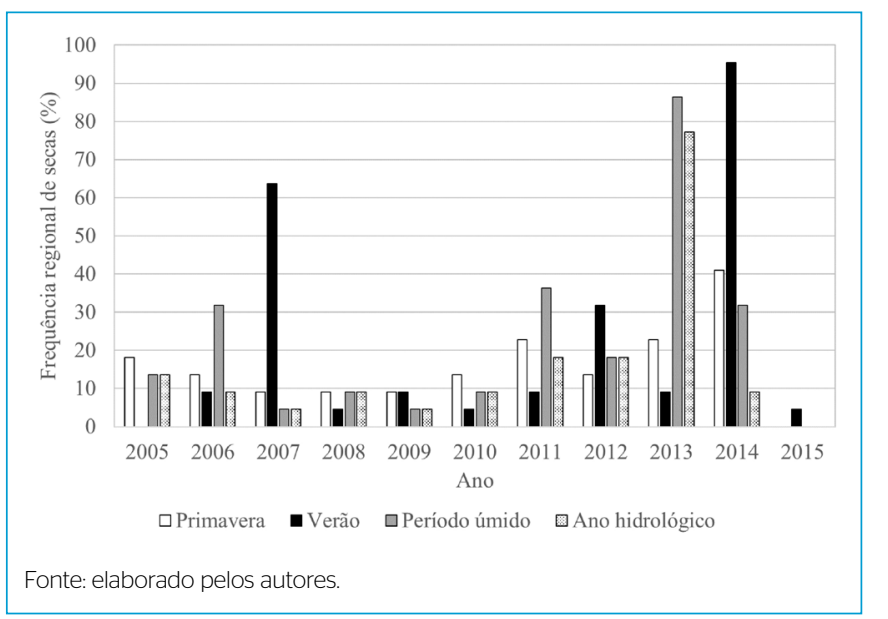

Figura 3 - Frequência regional de secas.

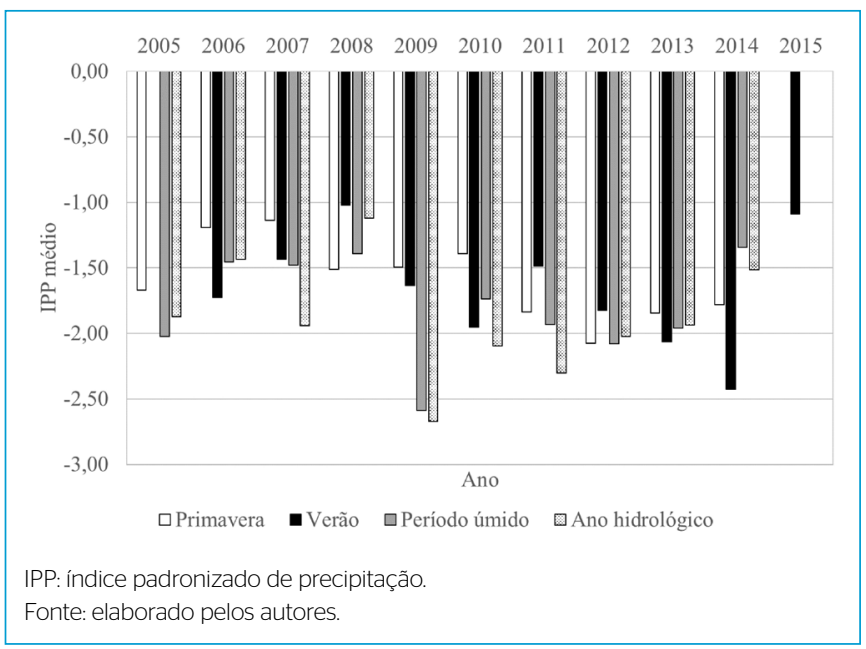

Figura 4 - Índices padronizados de precipitação médios regionais (das estações que apresentaram secas) de 2005 a 2014/2015. estações pluviométricas que apresentaram secas (IPP $\leq-1$ ), também, para cada escala temporal analisada. Considerando a BHRPS na sua totalidade (análise regional), as Figuras 3 e 4 mostram que o período úmido e o ano hidrológico de 2013/2014 foram severamente secos. Percebe-se, também, que o verão de 2014 foi o principal causador da crise hídrica na região, durante o qual mais de 90\% das estações pluviométricas apresentaram IPP médio (calculado a partir da média aritmética dos IPPs de cada estação) de aproximadamente -2,5 (que representa seca extrema).

A Tabela 4 apresenta os períodos mais secos de cada estação pluviométrica, dentro da extensão completa das séries de dados. Verifica-se, nessa tabela, que o verão de 2014 foi o mais seco em mais de 50 anos em estações localizadas em Queluz, Cachoeira Paulista, Lorena e Pindamonhangaba. Ainda segundo a Tabela 4, o ano de 1981 também foi um ano bastante seco na SPBHRPS. O período de 12 meses que começou no início de 1981 foi o mais seco em $45 \%$ das estações analisadas.

As Tabelas de 5 a 8 apresentam os IPPs para cada estação analisada e para todas as escalas temporais consideradas, entre os anos de 2005 a 2014. Essas tabelas possibilitam a análise dos padrões espaciais do IPP na região, além de sua variabilidade temporal no período mais recente. As Tabelas de 5 a 8 permitem concluir que a estação de Caçapava (código 2345008) foi a mais afetada pelos eventos de seca no período de 2005 a 2014, seguida pela estação de Aparecida (código 2245053). Esse fato é bastante interessante do ponto de vista espacial, uma vez que essas cidades/estações distam uma da outra aproximadamente $50 \mathrm{~km}$, e diversas cidades localizadas entre elas (por exemplo, Pindamonhangaba e Taubaté) não apresentaram padrões similares do IPP. Além disso, a estação 2345004, também localizada em Caçapava, apresentou um comportamento do IPP totalmente diferente da estação 2345008 . Tais resultados indicam a existência de condições microclimáticas bastante distintas no Vale do Rio Paraíba do Sul, cujas causas podem ser exploradas em futuros trabalhos. A estação de Santa Isabel (código 2346019) foi a menos afetada por secas entre 2005 e 2015, sendo essa a estação mais ocidental dentre as analisadas.

A Figura 5 apresenta o IPP médio das 22 estações pluviométricas analisadas - aqui, considerado o IPP regional da SPBHRPS - , para o período entre 2005 e 2014/2015. De acordo com a Figura 5, na escala de 12 meses, o principal período de seca que desencadeou a crise hídrica de 2014/2015 se iniciou em janeiro de 2013. Essa foi a seca mais intensa e longa durante os 10 anos anteriores a 2015. Considerando a operação do SHRPS, outro período de seca que contribui diretamente para a crise hídrica se iniciou em janeiro de 2011, tendo terminado em março de 2012. Embora mais curto e menos severo que a seca iniciada em 2013, a seca de 2011/2012 contribuiu com o agravamento da crise hídrica de 2014/2015, uma vez que a reduzida reposição do volume dos reservatórios do sistema causou um efeito cumulativo nos próximos anos hidrológicos. Isso pode ser claramente percebido na Figura 6, que apresenta simultaneamente a variabilidade temporal do IPP médio das estações estudadas (na escala de 12 meses) e o nível de armazenamento médio mensal do Reservatório de Paraibuna. A Figura 6 demonstra forte tendência de deplecionamento do armazenamento de Paraibuna a partir do início de 2011, que terminou no final de 2012, quando o volume armazenado atingiu $35 \%$ da capacidade do reservatório. Mesmo com significativa recuperação do volume durante o verão de 2012/2013, o Reservatório de Paraibuna atingiu apenas aproximadamente $65 \%$ de sua capacidade, cenário esse que foi precedido pela seca histórica de 2013/2014. 
Tabela 4 - Períodos mais secos históricos (mês e ano de início) das séries estudadas.

\begin{tabular}{|c|c|c|c|c|c|c|}
\hline $\begin{array}{l}\text { Código } \\
\text { ANA }\end{array}$ & Cidade & $\begin{array}{l}\text { Ano inicial } \\
\text { da série }\end{array}$ & $\begin{array}{l}\text { Mês mais } \\
\text { seco }\end{array}$ & $\begin{array}{l}\text { Três meses } \\
\text { mais secos }\end{array}$ & $\begin{array}{l}\text { Seis meses } \\
\text { mais secos }\end{array}$ & $\begin{array}{c}\text { Doze meses } \\
\text { mais secos }\end{array}$ \\
\hline 2244001 & Queluz & 1957 & out./05 & jan./14 & abr./63 & jan./14 \\
\hline 2244010 & Cachoeira Paulista & 1936 & fev./84 & jan./14 & jan./14 & dez./13 \\
\hline 2245020 & Lorena & 1957 & fev./84 & jan./14 & ago./81 & jan./81 \\
\hline 2245032 & Guaratinguetá & 1930 & jan./75 & jan./75 & nov./74 & ago./74 \\
\hline 2245035 & Pindamonhangaba & 1957 & $\mathrm{jan} / 07$ & jan./14 & nov./91 & jan./81 \\
\hline 2245048 & Pindamonhangaba & 1932 & jan./56 & jan./14 & mar./63 & jan./14 \\
\hline 2245053 & Aparecida & 1957 & jan./14 & nov./12 & set./12 & jan./81 \\
\hline 2245054 & Monteiro Lobato & 1939 & fev./57 & nov./92 & set./92 & $\mathrm{fev} / / 81$ \\
\hline 2345001 & Taubaté & 1963 & fev./73 & dez./72 & ago./81 & mar./81 \\
\hline 2345004 & Caçapava & 1969 & mar./15 & jul./11 & jun./10 & out./OO \\
\hline 2345008 & Caçapava & 1945 & jan./55 & out./81 & jul./81 & maio/11 \\
\hline 2345012 & Caçapava & 1942 & fev./84 & fev./81 & ago./81 & jan./81 \\
\hline 2345022 & Jambeiro & 1943 & jan./71 & jan./69 & jan./69 & $\mathrm{fev} . / 81$ \\
\hline 2345062 & Taubaté & 1937 & mar./68 & nov./05 & mar./68 & nov./79 \\
\hline 2345063 & Caçapava & 1929 & $\mathrm{fev} / \mathrm{77}$ & dez./13 & dez./13 & ago./13 \\
\hline 2345071 & Santa Branca & 1957 & out./14 & jul./63 & mar./75 & maio/77 \\
\hline 2345106 & Jacareí & 1960 & nov./96 & abr./00 & mar./75 & nov./13 \\
\hline 2345159 & Taubaté & 1937 & dez./81 & out./81 & jul./81 & jan./81 \\
\hline 2345161 & Taubaté & 1972 & out./89 & maio/63 & abr./63 & set./90 \\
\hline 2345164 & São José dos Campos & 1957 & fev./84 & fev./81 & ago./81 & jan./81 \\
\hline 2346019 & Santa Isabel & 1936 & abr./78 & out./37 & abr./63 & jun./44 \\
\hline 2346344 & Igaratá & 1957 & jan./75 & fev./81 & fev./81 & jan./81 \\
\hline
\end{tabular}

Os períodos marcados em cinza são diretamente associados à seca de 2013 a 2015.

Fonte: elaborado pelos autores.

Tabela 5 - Análise de secas (valores de IPP $\leq-1$ ) durante a primavera.

\begin{tabular}{|c|c|c|c|c|c|c|c|c|c|c|c|c|}
\hline \multirow{2}{*}{$\begin{array}{l}\text { Código } \\
\text { ANA }\end{array}$} & \multirow{2}{*}{ Cidade } & \multicolumn{10}{|c|}{ Ano } & \multirow{2}{*}{$\begin{array}{l}\text { Eventos } \\
\text { de seca }\end{array}$} \\
\hline & & 2005 & 2006 & 2007 & 2008 & 2009 & 2010 & 2011 & 2012 & 2013 & 2014 & \\
\hline 2244001 & Queluz & $-2,16$ & & & & & & & & & & 1 \\
\hline 2244010 & Cachoeira Paulista & & $-1,04$ & & & & & & & $-4,08$ & & 2 \\
\hline 2245020 & Lorena & & & & & & & & & & $-1,59$ & 1 \\
\hline 2245032 & Guaratinguetá & & & & & & & & & & $-1,78$ & 1 \\
\hline 2245035 & Pindamonhangaba & & & & & & & & & & $-1,54$ & 1 \\
\hline 2245048 & Pindamonhangaba & & & & & & & & & & $-2,31$ & 1 \\
\hline 2245053 & Aparecida & $-1,29$ & $-1,24$ & & & & & $-2,05$ & $-2,11$ & & & 4 \\
\hline 2245054 & Monteiro Lobato & & & & & & & & & & $-1,03$ & 1 \\
\hline 2345001 & Taubaté & $-1,18$ & & & & & & & & & $-1,42$ & 2 \\
\hline 2345004 & Caçapava & & & & & $-1,10$ & & $-1,47$ & & & & 2 \\
\hline 2345008 & Caçapava & & $-1,29$ & $-1,22$ & & $-1,89$ & $-1,63$ & $-1,94$ & $-2,72$ & & & 6 \\
\hline
\end{tabular}


Tabela 5 - Continuação.

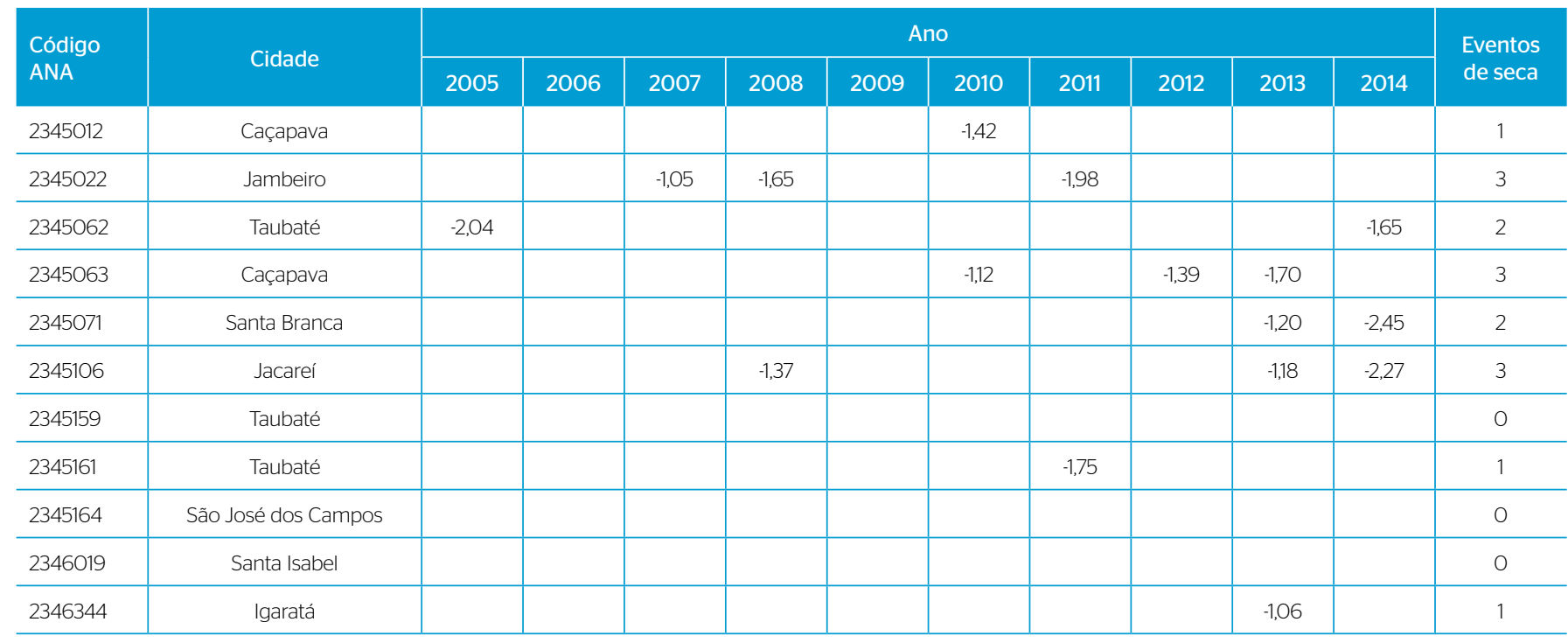

IPP: índice padronizado de precipitação.

Fonte: elaborado pelos autores.

Tabela 6 - Análise de secas (valores de IPP $\leq-1$ ) durante o verão.

\begin{tabular}{|c|c|c|c|c|c|c|c|c|c|c|c|c|}
\hline $\begin{array}{l}\text { Código } \\
\text { ANA }\end{array}$ & Cidade & \multicolumn{10}{|c|}{ Ano } & $\begin{array}{l}\text { Eventos } \\
\text { de seca }\end{array}$ \\
\hline 2244001 & Queluz & & & & & & & $-2,20$ & & $-2,92$ & & 2 \\
\hline 2244010 & Cachoeira Paulista & & $-1,04$ & & & & & & & $-4,08$ & & 2 \\
\hline 2245020 & Lorena & & $-1,08$ & & & & & & & $-3,45$ & & 2 \\
\hline 2245048 & Pindamonhangaba & & $-1,21$ & & & & & $-1,36$ & & $-3,56$ & & 3 \\
\hline 2245053 & Aparecida & $-1,72$ & & & & & $-1,43$ & $-2,74$ & $-2,18$ & $-3,15$ & & 5 \\
\hline 2245054 & Monteiro Lobato & & & & & & & & & $-2,25$ & & 1 \\
\hline 2345001 & Taubaté & & $-1,18$ & & & & & & & $-1,37$ & $-1,09$ & 3 \\
\hline 2345012 & Caçapava & & $-1,26$ & & & & & & & $-2,08$ & & 2 \\
\hline 2345022 & Jambeiro & & & $-1,02$ & & & & $-1,16$ & & $-1,63$ & & 3 \\
\hline 2345062 & Taubaté & $-1,73$ & $-1,01$ & & & & & & & $-1,28$ & & 3 \\
\hline 2345063 & Caçapava & & $-1,49$ & & & & & & & $-4,25$ & & 2 \\
\hline 2345071 & Santa Branca & & & & & & & & & $-1,65$ & & 1 \\
\hline 2345106 & Jacareí & & $-1,98$ & & $-1,18$ & & & & & $-1,98$ & & 3 \\
\hline 2345159 & Taubaté & & $-1,35$ & & & & & & & $-1,98$ & & 2 \\
\hline 2345161 & Taubaté & & & & & & & $-1,70$ & & & & 1 \\
\hline 2345164 & São José dos Campos & & $-1,44$ & & & & & & & $-1,89$ & & 2 \\
\hline
\end{tabular}

IPP: índice padronizado de precipitação. 
Tabela 7 - Análise de secas (valores de IPP $\leq-1$ ) durante o período úmido.

\begin{tabular}{|c|c|c|c|c|c|c|c|c|c|c|c|c|}
\hline \multirow{2}{*}{$\begin{array}{l}\text { Código } \\
\text { ANA }\end{array}$} & \multirow[b]{2}{*}{ Cidade } & \multicolumn{10}{|c|}{ Ano } & \multirow{2}{*}{$\begin{array}{l}\text { Eventos } \\
\text { de seca }\end{array}$} \\
\hline & & $\begin{array}{l}2005 \\
2006\end{array}$ & $\begin{array}{l}2006 \\
2007\end{array}$ & $\begin{array}{l}2007 \\
2008\end{array}$ & $\begin{array}{l}2008 \\
2009\end{array}$ & $\begin{array}{l}2009 \\
2010\end{array}$ & $\begin{array}{r}2010 \\
2011\end{array}$ & $\begin{array}{l}2011 \\
2012\end{array}$ & $\begin{array}{l}2012 \\
2013\end{array}$ & $\begin{array}{l}2013 \\
2014\end{array}$ & $\begin{array}{l}2014 \\
2015\end{array}$ & \\
\hline 2244001 & Queluz & $-1,71$ & & & & & & $-1,23$ & & $-1,95$ & & 3 \\
\hline 2244010 & Cachoeira Paulista & & & & & & & & & $-3,48$ & & 1 \\
\hline 2245020 & Lorena & & & & & & & & $-1,03$ & $-2,65$ & $-1,16$ & 3 \\
\hline 2245032 & Guaratinguetá & & & & & & & & & $-1,81$ & $-1,24$ & 2 \\
\hline 2245035 & Pindamonhangaba & & $-1,22$ & & & & & $-1,17$ & & $-1,64$ & $-1,06$ & 4 \\
\hline 2245048 & Pindamonhangaba & & $-1,25$ & & & & & $-1,61$ & & $-2,33$ & $-1,45$ & 4 \\
\hline 2245053 & Aparecida & $-1,79$ & $-1,17$ & & & & $-1,33$ & $-3,12$ & $-3,07$ & $-2,24$ & & 6 \\
\hline 2245054 & Monteiro Lobato & & & & & & & & & $-1,26$ & & 1 \\
\hline 2345001 & Taubaté & & & & & & & & & $-1,07$ & $-1,67$ & 2 \\
\hline 2345004 & Caçapava & & & & & & & $-1,06$ & & & & 1 \\
\hline 2345008 & Caçapava & & $-2,24$ & & $-1,01$ & $-2,59$ & $-2,14$ & $-3,04$ & $-2,99$ & $-2,05$ & & 7 \\
\hline 2345012 & Caçapava & & $-1,20$ & & & & & & & & & 1 \\
\hline 2345022 & Jambeiro & & & $-1,48$ & & & & $-2,20$ & & $-1,15$ & & 3 \\
\hline 2345062 & Taubaté & $-2,57$ & & & & & & & & $-1,05$ & $-1,74$ & 3 \\
\hline 2345063 & Caçapava & & $-1,73$ & & & & & & & $-4,33$ & & 2 \\
\hline 2345071 & Santa Branca & & & & & & & & & $-1,90$ & & 1 \\
\hline 2345106 & Jacareí & & & & $-1,77$ & & & & $-1,22$ & $-2,25$ & $-1,09$ & 4 \\
\hline 2345159 & Taubaté & & & & & & & & & $-1,77$ & & 1 \\
\hline 2345161 & Taubaté & & & & & & & $-2,01$ & & & & 1 \\
\hline 2345164 & São José dos Campos & & $-1,37$ & & & & & & & $-1,27$ & & 2 \\
\hline 2346019 & Santa Isabel & & & & & & & & & $-1,15$ & & 1 \\
\hline 2346344 & Igaratá & & & & & & & & & $-1,87$ & & 1 \\
\hline
\end{tabular}

IPP: índice padronizado de precipitação.

Tabela 8 - Análise de secas (valores de IPP $\leq-1$ ) durante o ano hidrológico.

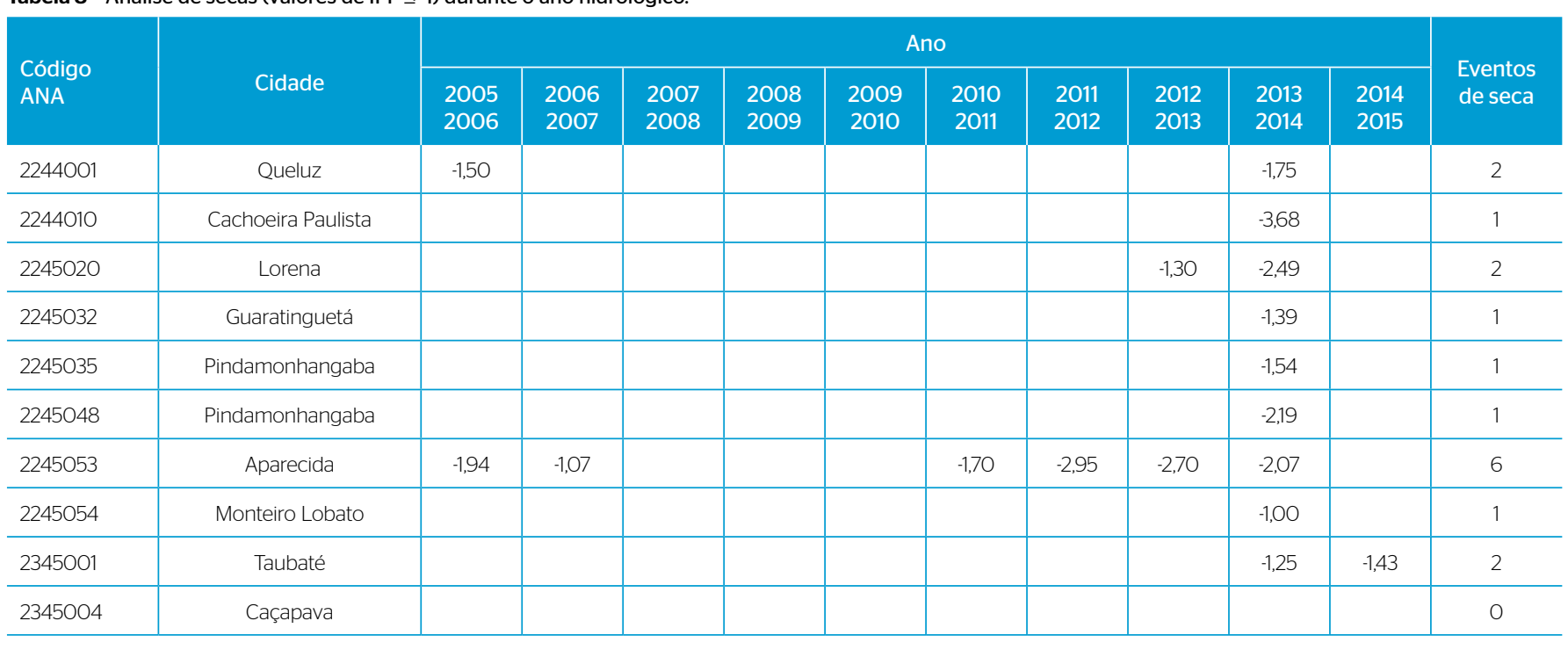


Tabela 8 - Continuação.

\begin{tabular}{|c|c|c|c|c|c|c|c|c|c|c|c|c|}
\hline \multirow{2}{*}{$\begin{array}{l}\text { Código } \\
\text { ANA }\end{array}$} & \multirow[b]{2}{*}{ Cidade } & \multicolumn{10}{|c|}{ Ano } & \multirow{2}{*}{$\begin{array}{l}\text { Eventos } \\
\text { de seca }\end{array}$} \\
\hline & & $\begin{array}{l}2005 \\
2006\end{array}$ & $\begin{array}{l}2006 \\
2007\end{array}$ & $\begin{array}{l}2007 \\
2008\end{array}$ & $\begin{array}{l}2008 \\
2009\end{array}$ & $\begin{array}{l}2009 \\
2010\end{array}$ & $\begin{array}{l}2010 \\
2011\end{array}$ & $\begin{array}{l}2011 \\
2012\end{array}$ & $\begin{array}{l}2012 \\
2013\end{array}$ & $\begin{array}{l}2013 \\
2014\end{array}$ & $\begin{array}{l}2014 \\
2015\end{array}$ & \\
\hline 2345008 & Caçapava & & $-1,80$ & & $-1,24$ & $-2,67$ & $-2,49$ & $-2,91$ & $-2,97$ & $-1,96$ & & 7 \\
\hline 2345012 & Caçapava & & & & & & & & & & & 0 \\
\hline 2345022 & Jambeiro & & & $-1,94$ & & & & $-1,73$ & & & & 2 \\
\hline 2345062 & Taubaté & $-2,18$ & & & & & & & & $-1,32$ & $-1,60$ & 3 \\
\hline 2345063 & Caçapava & & & & & & & & & $-3,86$ & & 1 \\
\hline 2345071 & Santa Branca & & & & & & & & & $-1,82$ & & 1 \\
\hline 2345106 & Jacareí & & & & $-1,00$ & & & & $-1,13$ & $-2,02$ & & 3 \\
\hline 2345159 & Taubaté & & & & & & & & & $-1,70$ & & 1 \\
\hline 2345161 & Taubaté & & & & & & & $-1,61$ & & & & 1 \\
\hline 2345164 & São José dos Campos & & & & & & & & & $-1,21$ & & 1 \\
\hline 2346019 & Santa Isabel & & & & & & & & & & & 0 \\
\hline 2346344 & Igaratá & & & & & & & & & $-1,69$ & & 1 \\
\hline
\end{tabular}

IPP: índice padronizado de precipitação.

Fonte: elaborado pelos autores.

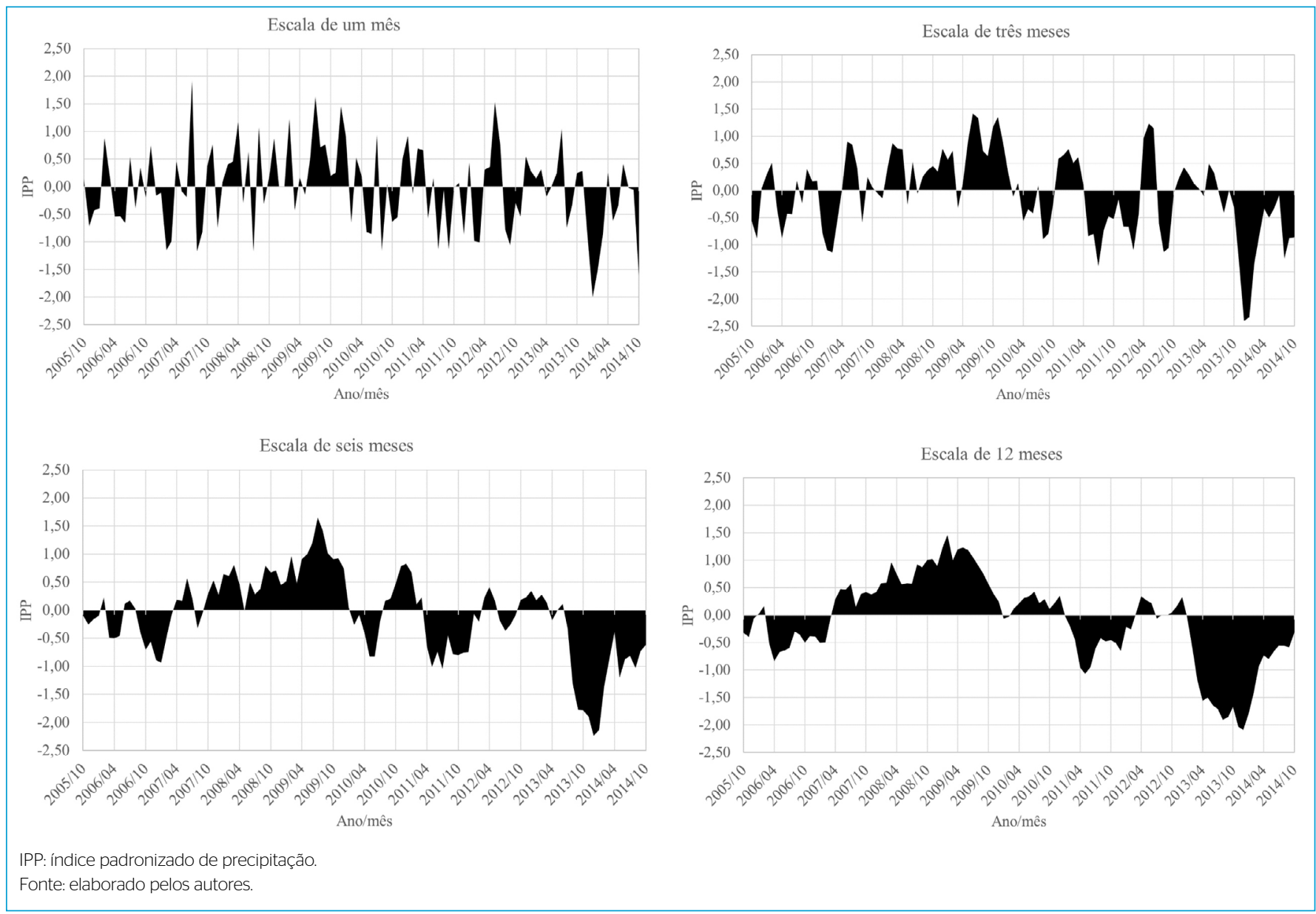

Figura 5 - Índice padronizado de precipitação médio regional entre 2005 e 2014/2015. 


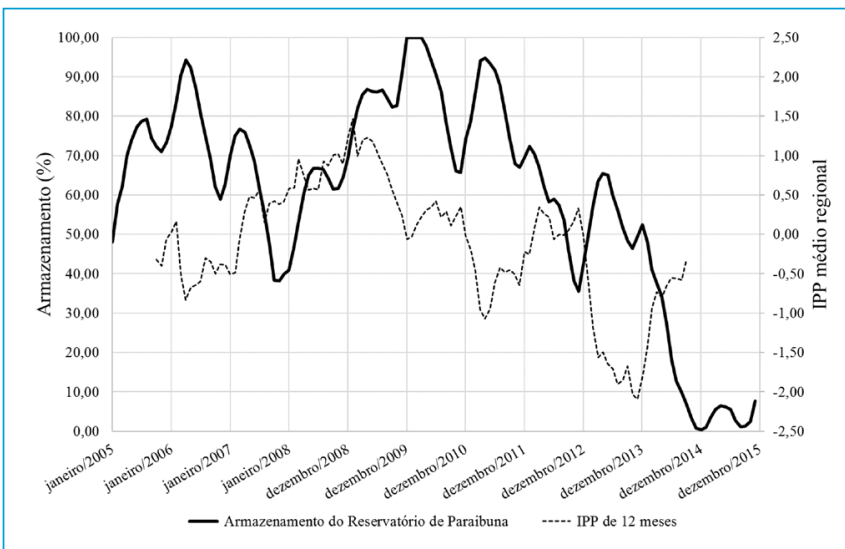

IPP: índice padronizado de precipitação.

Fonte: elaborado pelos autores.

Figura 6 - Variabilidade do armazenamento do Reservatório de Paraibuna (valores médios mensais) e do índice padronizado de precipitação médio das estações estudadas (na escala de 12 meses).

Segundo Coelho et al. (2016), a seca da Região Sudeste do Brasil durante o verão (janeiro a março) de 2014 foi decorrente de teleconexões geradas a partir de uma fonte de calor tropical anômala no norte/nordeste da Austrália. Em termos climatológicos, alguns trabalhos analisaram os padrões de circulação atmosférica de grande escala que resultaram no período de seca/crise hídrica estudado no presente trabalho. Rodrigues et al. (2019), por sua vez, investigaram as causas comuns da seca de 2013/2014 e de ondas de calor marítimas nesse meio período, tendo concluído que tais eventos foram decorrentes de um bloqueio atmosférico desencadeado por convecção tropical nos oceanos Índico e Pacífico.

\section{CONCLUSÕES}

O verão de 2014 foi o período crítico que desencadeou o pico da crise hídrica que afetou a SPBHRPS durante a primeira metade da década de 2010. Em mais de $90 \%$ das estações pluviométricas analisadas, a quantidade de chuva durante o verão de 2014 foi classificada como seca extrema, a partir dos padrões do IPP. Por consequência do verão de 2014, o período úmido e o ano hidrológico de 2013/2014 foram igualmente secos.
O verão de 2014 foi o mais seco de todo o histórico de dados de estações pluviométricas localizadas na região norte da SPBHRPS. Essa região se localiza entre os municípios de Pindamonhangaba, Lorena, Cachoeira Paulista e Queluz, demonstrando um padrão geográfico em relação à seca. Considerando a escala de 12 meses, estações localizadas em Queluz, Cachoeira Paulista, Lorena, Pindamonhangaba, Aparecida, Caçapava, Santa Branca e Jacareí apresentaram seus períodos de 12 meses mais secos, em termos históricos, durante o evento da década de 2010.

O pico da crise hídrica entre 2014 e 2015 ocorreu em razão do período úmido 2013/2014 (no qual ocorreu o pico da seca), durante o qual quase $90 \%$ das estações pluviométricas estudadas apresentou IPPs de seca, e o IPP regional se aproximou do valor de $-2,00$, que classifica esse período como de seca severa.

$\mathrm{O}$ ano de 2007 também apresentou um verão seco em mais de 60\% das estações pluviométricas, mas com intensidade menor (IPP médio pouco superior a -1,5, indicando seca moderada). Junto à primavera seca de 2006, em algumas das estações, esse cenário resultou em um ano hidrológico seco em 2006/2007. Esse evento de seca levou o armazenamento do Reservatório de Paraibuna a aproximadamente 35\% (Figura 1), mas não influenciou a crise hídrica da década de 2010. Como pode ser visto na Figura 1, o volume útil do Reservatório de Paraibuna foi completamente recuperado nos anos após 2007 (em razão, principalmente, de um período úmido entre 2008 e 2009), antes da ocorrência da seca de 2013/2014.

Embora o pico da seca tenha ocorrido em 2014, a crise hídrica na BHRPS foi resultado de um efeito cumulativo de uma seca anterior, que começou no início de 2011. Esse evento de seca anterior levou o Reservatório de Paraibuna a baixos níveis de armazenamento, que não foram repostos antes do evento de seca ocorrido entre 2013 e 2015. Essa conclusão destaca a importância do planejamento e operação plurianuais do SHRPS, tendo em vista a propagação interanual dos efeitos da chuva detectada neste estudo. Nesse sentido, destaca-se, também, a importância da utilização de modelos hidrológicos e climáticos dentre as ferramentas de planejamento do SHRPS, de forma a se prever anomalias da precipitação e suas repercussões, permitindo o ajuste precoce das condições operacionais para a mitigação das consequências de tais anomalias.

\section{CONTRIBUIÇÕES DOS AUTORES}

Neves, A.: Curadoria de Dados, Análise Formal, Investigação. Vilanova, M.: Análise Formal, Investigação.

\section{REFERÊNCIAS}

ABRAMOWITZ, M.; STEGUN, I.A. Handbook of Mathematical Functions with Formulas, Graphs, and Mathematical Tables. Nova York: Dover, 1965.

AGÊNCIA NACIONAL DE ÁGUAS (ANA). Boletim de monitoramento dos reservatórios do Sistema Hidráulico do Rio Paraíba do Sul: maio de 2017. Brasília: ANA, 2017.

BONACCORSO, B.; PERES, D.J.; CASTANO, A.; CANCELLIERE, A. SPI-Based Probabilistic Analysis of Drought Areal Extent in Sicily. Water Resources
Management, v. 29, n. 2, p. 459-470, 2015. https://doi.org/10.1007/s11269-014 0673-4

BRASIL. Ministério da Integração Nacional. Anuário Brasileiro de Desastres Naturais 2013. Brasília: Ministério da Integração Nacional, 2014.

CALOIERO, T. Drought analysis in New Zealand using the standardized precipitation index. Environmental Earth Sciences, v. 76, n. 16, p. 569, 2017. https://doi.org/10.1007/s12665-017-6909-x 
CHOW, V.T.; MAIDMENT, D.R.; MAYS, L.W. Applied hydrology. [S.I.]: McGrawHill, 1988.

COELHO, C.A.S.; OLIVEIRA, C.P.; AMBRIZZI, T.; REBOITA, M.S.; CARPENEDO, C.B.; CAMPOS, J.L.P.S.; TOMAZIELLO, A.C.N.; PAMPUCH, L.A.; CUSTÓDIO, M.S.; DUTRA, L.M.M; ROCHA, R.P.; REHBEIN, A. The 2014 southeast Brazil austral summer drought: regional scale mechanisms and teleconnections. Climate Dynamics, v. 46, n. 11-12, p. 3737-3752, 15 jun. 2016. https://doi.org/10.1007/ s00382-015-2800-1

EDWARDS, D.C.; MCKEE, T.B. Characteristicas of 20th century drought in the United States at multiple time scales. Climatology report no. 97-2. Fort Collins: [s.n.], 1997.

ESCOBAR, H. Drought triggers alarms in Brazil's biggest metropolis. Science, v. 347, n. 6224, p. 812, 20 fev. 2015. https://doi.org/10.1126/science.347.6224.812

GOVERNO DO ESTADO DESÃO PAULO; FUNDO ESTADUAL DE RECURSOS HÍDRICOS; COMITE DAS BACIAS HIDROGRAFICAS DO RIO PARAIBAA DO SUL. UGRHI-O2: Relatório de situação 2016 - Ano base 2015. São Paulo: Comitê das Bacias Hidrográficas do Rio Paraíba do Sul, 2016

$\measuredangle A B E D Z K I, L$. Categorical forecast of precipitation anomaly using the standardized precipitation index SPI. Water, v. 9, n. 1, p. 8, 2017. https://doi. org/10.3390/w9010008

MCKEE, T.B.; DOESKEN, N.J.; KLEIST, J. The relationship of drought frequency and duration to time scales. In: CONFERENCE ON APPLIED CLIMATOLOGY, 8., 1993, Anaheim. Anais [...]. Anaheim: American Meteorological Society, 1993.

MISHRA, A.K.; SINGH, V.P. A review of drought concepts. Journal of Hydrology, v. 391, n. 1-2, p. 202-216, set. 2010. https://doi.org/10.1016/j. jhydrol.2010.07.012

NAGHETTINI, M.; PINTO, É.J.A. Hidrologia Estatística. Belo Horizonte: CPRM, 2007.

NEVES, A.O. Aplicação do índice padronizado de precipitação para a análise da seca 2014/2015 na porção Paulista da Bacia do Rio Paraíba do Sul. 150f.
Dissertação (Mestrado) - Faculdade de Engenharia de Guaratinguetá, Universidade Estadual Paulista “Júlio de Mesquita Filho”, Guaratinguetá, 2016.

RODRIGUES, R.R.; TASCHETTO, A.S.; GUPTA, A.S.; FOLTZ, G.R. Common cause for severe droughts in South America and marine heatwaves in the South Atlantic. Nature Geoscience, v. 12, n. 8, p. 620-626, 8 ago. 2019. https:// doi.org/10.1038/s41561-019-0393-8

SÃO PAULO. Secretaria do Meio Ambiente. Subsídios ao planejamento ambiental da unidade hidrográfica de gerenciamento de recursos hídricos Paraíba do Sul: UGRHI O2. São Paulo: Secretaria do Meio Ambiente, 2011.

SAYERS, P.B.; YUANYUAN, L.; MONCRIEFF, C.; JIANQIANG, L.; TICKNER D.; GANG, L.; SPEED, R. Strategic drought risk management: eight 'golden rules' to guide a sound approach. International Journal of River Basin Management, v. 15, n. 2, p. 239-255, 2017. https://doi.org/10.1080/15715124.2 017.1280812

SVOBODA, M.; HAYES, M.; WOOD, D.A.; WORLD METEOROLOGICAL ORGANIZATION. Standardized precipitation index: User guide. Genebra: World Meteorological Organization, 2012

TIGKAS, D.; VANGELIS, H.; TSAKIRIS, G. DrinC: a software for drought analysis based on drought indices. Earth Science Informatics, v. 8, n. 3, p. 697-709, 25 set. 2015. https://doi.org/10.1007/s12145-014-0178-y

TSAKIRIS, G.; VANGELIS, H. Towards a Drought Watch System based on Spatial SPI. Water Resources Management, v. 18, n. 1, p. 1-12, fev. 2004. https:// doi.org/10.1023/B:WARM.0000015410.47014.a4

WORLD METEOROLOGICAL ORGANIZATION; UNITED NATIONS EDUCATIONAL SCIENTIFIC AND CULTURAL ORGANIZATION. International Glossary of Hydrology. Genebra: WMO, 2012. Disponível em: http://www. wmo.int/pages/prog/hwrp/publications/international_glossary/385 IGH_2012.pdf. Acesso em: 31 dez. 2019.

YAN, Z:; ZHANG, Y.; ZHOU, Z:; HAN, N. The spatio-temporal variability of droughts using the standardized precipitation index in Yunnan, China. Natural Hazards, v. 88, n. 2, p. 1023-1042, 2017. https://doi.org/10.1007/s11069017-2904-5 